\title{
Menso Folkerts über Boethius, Gauß und die Zukunft des wissenschaftshistorischen Studiums
}

In diesem Jahr kann Prof. Dr. Menso Folkerts (Ludwig-Maximilians-Universität München), auf eine 40jährige erfolgreiche Tätigkeit in Forschung und Lehre in Berlin, Oldenburg und München zurückblicken. Seit vielen Jahren ist er als Autor und Rezensent für NTM tätig, war seit 1998 einer ihrer Herausgeber und gehört heute dem wissenschaftlichen Beirat an. Seine Versetzung in den Ruhestand bietet Anlass, nach seinen wichtigsten Lebensstationen und Forschungsgebieten zu fragen.

F: Im Jahr 1960 wurde die Zeitschrift NTM von Gerhard Harig und Alexander Mette gegründet. Nach 1989 geriet die Zeitschrift - nachdem sie anfänglich zu den früheren Bedingungen weitergeführt wurde - in Turbulenzen. Bei ihrer Rettung waren Sie maßgeblich beteiligt. Wie konnte NTM überleben?

A: Ich war eine von mehreren Personen, die sich in dieser Beziehung engagiert haben. Ganz wesentlich waren die Verhandlungen von Herrn Wußing mit dem Birkhäuser Verlag. Dazu kam, dass auch die anderen Herausgeber Kontakte zu Birkhäuser besaßen. Ich selbst hatte schon bei Birkhäuser publiziert und kannte deshalb die Personen, die mit den wissenschaftshistorischen Publikationen betraut waren. Meine Kontakte zu Hans Wußing und den anderen Herausgebern haben dazu beigetragen, dass ich hier aktiv werden konnte. Unsere gemeinsamen Bemühungen führten schließlich dazu, dass die Zeitschrift für einige Jahre auf eine solide finanzielle Basis gestellt werden konnte und dass dann mittelfristig und - wie wir jetzt wissen - auch langfristig das Überleben garantiert war.

F: Sie feiern in diesem Jahr Ihren 65. Geburtstag. Wenn Sie zurückschauen: Welche Personen sind für Ihr wissenschaftliches Leben prägend gewesen?

A: Da muss ich beim Studium beginnen. Ich habe Klassische Philologie und Mathematik in Göttingen studiert, zwei Fächer, die natürlich sehr unterschiedlich sind, und es war relativ ungewöhnlich, eine solche Kombination zu wählen. Die Mathematiker in Göttingen hatten an der Geschichte ihres 
Faches naturgemäß wenig Interesse, und die Altphilologen hatten eine gewisse Scheu vor der Mathematik. Aber ich hatte das Glück, mit Jürgen Mau einen Privatdozenten zu treffen, der an der Berliner Akademie gearbeitet hatte und nach dem Mauerbau nach Göttingen kam. Mau war ein Fachmann für griechische Philosophie und Mathematik und bot in Göttingen im „Seminar für Klassische Philologie" eine Veranstaltung an - die Lektüre mathematischer griechischer Texte -, die von drei Zuhörern besucht wurde und in der Originaltexte von Euklid, Archimedes und anderen Autoren gelesen wurden. Dies war für mich so etwas wie ein zentrales Erlebnis, weil ich zum ersten Mal die Möglichkeit hatte, mathematische Texte aus der Antike im Original zu lesen, zu verstehen und ihre besondere Problematik kennenzulernen. Das war der Anlass, mich in der Folgezeit näher mit der Geschichte der Mathematik zu beschäftigen, die mich schon immer interessiert hatte. Weiterhin war für mich Professor Götting wichtig, der kurz zuvor in Göttingen den Lehrstuhl für historische Hilfswissenschaften erhalten hatte. Durch ihn habe ich anhand von Original-Handschriften die lateinische Paläographie des Mittelalters lernen können. Diese beiden Personen haben mich dann dazu gebracht, mich näher mit der Überlieferung antiker mathematischer Texte zu beschäftigen und aus diesem Gebiet ein Thema für meine Dissertation zu wählen: die Edition einer mathematischen Schrift, die Boethius zugeschrieben wurde, aber in Wirklichkeit im 11. Jahrhundert entstanden ist. Ich habe sozusagen autodidaktisch, ohne Unterstützung durch ein normales Studium der Wissenschaftsgeschichte, mir die Kenntnisse erworben, die man für derartige Arbeiten braucht. Dann hatte ich das Glück, dass meine Arbeit von einem Gräzisten, Karl Deichgräber, als Dissertation angenommen wurde, so dass ich damit 1967 promovieren konnte. Durch diese Arbeit wurde ich in den Kreis der Historiker der Mathematik eingeführt. Im Wesentlichen geschah das dadurch, dass ich nach München an das Institut für Geschichte der Naturwissenschaften eingeladen wurde, um über meine Arbeit vorzutragen, und auch an das entsprechende Institut in Hamburg. In München war Kurt Vogel tätig, der zwar nicht die Professur für Wissenschaftsgeschichte innehatte, aber doch seit den 1930er Jahren im Bereich der Mathematikgeschichte lehrte und forschte. Der Lehrstuhlinhaber war Helmuth Gericke. Von beiden habe ich viele Dinge erfahren, die mir noch nicht vertraut waren, weil ich keine wissenschaftshistorische Vorbildung besaß. Hier ist vor allem Kurt Vogel prägend für mich geworden, weil sein Arbeitsschwerpunkt die Mathematik im Altertum, im Mittelalter und in der frühen Neuzeit war. Ein Glücksfall war es wiederum, dass ich später als Nachfolger von Helmuth Gericke in München (seit 1980) auf diesem Gebiet tätig sein konnte und noch die Gelegenheit hatte, direkt mit Kurt Vogel zusammenzuarbeiten. Er ist eine ganz entscheidende Person für meinen wissenschaftlichen Werdegang gewesen. Schließlich sollte ich noch erwähnen, dass ich durch meine Dissertation und durch die Vermittlung von Gericke auch mit Joseph Ehrenfried Hofmann in 
Kontakt kam. Er ist in Deutschland und weit darüber hinaus die entscheidende Person in der Mathematikgeschichte der 1960er Jahre gewesen, weil er regelmäßig Tagungen zur Geschichte der Mathematik im Mathematischen Forschungsinstitut Oberwolfach geleitet hat. Ich wurde dazu eingeladen und kam durch ihn mit anderen Mathematikhistorikern in Kontakt.

F: Wo begann ihre berufliche Laufbahn in der Wissenschaftsgeschichte?

A: Meine Oberwolfacher Kontakte führten dazu, dass ich meine erste Stelle in diesem Bereich bekam, weil nämlich ein Schüler von Hofmann, Christoph Scriba, gerade an die TU Berlin berufen worden war und mich fragte, ob ich bereit wäre, als sein Assistent zu arbeiten. Die Zeit von 1969 bis 1976, in der ich in Berlin gelebt habe, war sicher prägend für mich, besonders die Zusammenarbeit mit Christoph Scriba, der mich in einer ganz uneigennützigen Art gefördert und meinen Bildungshorizont in der Wissenschaftsgeschichte erweitert hat. Ich habe gemeinsam mit ihm und mit dem Technikhistoriker Kurt Mauel die Bibliothek des Lehrstuhls für Geschichte der exakten Wissenschaften und der Technik aufgebaut, für die viel Geld zur Verfügung stand. Bei der Literaturbeschaffung habe ich sehr viel gelernt und ich bin Herrn Scriba sehr dankbar dafür, dass er sich viel Zeit nahm, um meine Literaturvorschläge durchzusehen und gegebenenfalls zu korrigieren. In dieser Zeit konnte ich das nachholen, was man heute als Student der Geschichte der Naturwissenschaften lernen kann. Ich habe mich dann in Berlin habilitiert - zu einer Zeit, als das Fach noch aufstrebend war.

F: Die längste Zeit Ihrer wissenschaftlichen Laufbahn haben Sie als Lehrstuhlinhaber und Leiter des Instituts für Geschichte der Naturwissenschaften in München verbracht. Was war in München anders?

A: Zunächst einmal war München, verglichen mit Berlin, ein Ort, an dem die Geschichte der Naturwissenschaften durch ein Institut etabliert war, das schon 1963 eingerichtet worden ist - gemeinsam mit zwei weiteren Zentren an der Technischen Universität und am Deutschen Museum. Damals war München das Zentrum der Wissenschaftsgeschichte in Deutschland. Bevor ich nach München kam, war ich vier Jahre lang Professor an der Universität Oldenburg gewesen. Dort hatte ich eine Professur für Mathematik mit dem Schwerpunkt Berufspraxis und Geschichte der Mathematik, das heißt, ich sollte den künftigen Mathematiklehrern Kenntnisse in der Geschichte vermitteln und andererseits den künftigen Diplom-Mathematikern Kenntnisse über ihre Berufspraxis. Dies war eine neue berufliche Herausforderung für mich. Im Übrigen war Oldenburg eine Reform-Universität, an der die einphasige Lehrerausbildung ausgeübt wurde, das heißt, die didaktischen Kenntnisse, die traditionsgemäß in der Referendarzeit erworben werden, wurden schon während des Studiums vermittelt. In Oldenburg war ich der einzige Wissenschaftshistoriker innerhalb von zahlreichen Mathematikern 
und Didaktikern. Die Politisierung der Hochschule war in Oldenburg stark spürbar, während die Ludwig-Maximilians-Universität in München eine traditionelle Universität war, die viele Möglichkeiten bot, sich der Geschichte der Naturwissenschaften zu widmen.

F: Heute sind die Aussichten für die Wissenschaftsgeschichte in München weniger glänzend. Es war bereits geplant, sie ganz aus dem Curriculum der Ludwig-Maximilians-Universität zu verbannen. Wie sehen Sie heute die Zukunftsaussichten des Faches, und wie wichtig ist die Wissenschaftsgeschichte aus Ihrer Sicht für die zukünftige Entwicklung der Universität?

A: Zunächst einmal sollte man hervorheben, dass lange Jahre hindurch das Fach Geschichte der Naturwissenschaften in keiner Weise gefährdet war. Im Gegenteil: In den 1980er und frühen 1990er Jahren blühte es auf. Es gab eine kritische Masse in München, vereinigt in den Räumen des Deutschen Museums. Die Kollegen an den verschiedenen Institutionen organisierten gemeinsame Veranstaltungen. Die Probleme entstanden erst gegen Ende der 1990er Jahre in Verbindung mit dem knapper werdenden Geld. Man fragte sich, ob man sich solche „Orchideenfächer" wie die Geschichte der Naturwissenschaften noch weiterhin leisten könne. Freiwerdende Stellen sollten nicht wieder besetzt werden. In München wurde die Situation durch die Umstrukturierung der Universität weiter erschwert, da eine Mindestanzahl von Hochschullehrern pro Institut vorgeschrieben war, so dass kleine Institute nicht weiter bestehen konnten. Dies führte dazu, dass das Institut, das früher an der Mathematischen Fakultät angesiedelt war, als Abteilung in das Historische Seminar integriert wurde. Die Wissenschaftsgeschichte hatte schon immer gewisse Probleme, weil sie weder von den Naturwissenschaftlern noch von den Historikern als gleichberechtigt anerkannt wurde. So wurden die ursprünglichen drei Professuren, die es an der LMU München gab, im Laufe der Zeit durch Universitätswechsel und Pensionierung auf eine Stelle reduziert. Ebenso wie bei der Universitäts- und Bildungsgeschichte wurde die Notwendigkeit der Geschichte der Naturwissenschaften an der Münchner Universität generell in Frage gestellt. Das führte zu vielen nationalen und internationalen Protestschreiben. Schließlich gab es zwei voneinander unabhängige Evaluierungen, in denen detailliert empfohlen wurde, das Fach Geschichte der Naturwissenschaften und der Technik nicht nur nicht einzustellen, sondern sogar weiter auszubauen. Das hat beim Bayerischen Staatsministerium für Bildung, Forschung und Kunst zu einem gewissen Umdenken geführt, aber an der Universität noch keine Planungssicherheit hergestellt, sodass heute, ein paar Monate vor meinem Eintritt in den Ruhestand, noch immer nicht klar ist, ob meine Stelle wieder besetzt werden kann.

F: Der Name Boethius im Zusammenhang mit der Euklid-Rezeption bezeichnet ein zentrales Thema Ihrer Forschungstätigkeit. Hat Boethius für Sie heute 
noch den gleichen Stellenwert wie in den vergangenen Jahrzehnten? Hinzugekommen ist die Gauß-Forschung, die heute vielleicht mehr im Mittelpunkt steht. Welchen Forschungsschwerpunkten werden Sie sich nach dem Eintritt in den Ruhestand vorrangig widmen?

A: Der Name Boethius besitzt für mich zwei Aspekte: Zum einen bezeichnet er den Autor, über den ich promoviert habe, dann aber auch eine wissenschaftshistorische Reihe, die schon existierte, bevor ich promovierte und die ich später als Herausgeber übernehmen konnte. Inzwischen ist Boethius zu einer der führenden wissenschaftshistorischen Reihen in Deutschland geworden. Zugleich ist Boethius für mich ein Synonym für meinen ursprünglichen Forschungsschwerpunkt, nämlich die Erforschung der Geschichte der Mathematik und der mathematischen Naturwissenschaften im Mittelalter. Meine Arbeit auf diesem Gebiet dauert bis heute an, wobei ich das Interesse ein wenig ausgebaut habe - bis in die frühe Neuzeit hinein. Derzeit bin ich intensiv damit beschäftigt, ein „Handbuch zur Geschichte der Mathematik im Mittelalter" fertig zu stellen, das ich schon vor Jahren gemeinsam mit meinem Kollegen und Freund H. L. L. Busard, der leider vor kurzem gestorben ist, begonnen habe. Das Ziel ist eine umfangreiche Darstellung der Geschichte der Mathematik im westlichen Mittelalter, beginnend mit der Zeit von Boethius und endend mit der Zeit um 1500. Neben einem Überblick über die historische Entwicklung wird auch die spezielle Leistung der einzelnen Personen auf diesem Gebiet gewürdigt. Im Übrigen habe ich vor kurzem die Herausgabe der mathematischen Schriften von Nikolaus von Kues abgeschlossen - das führt in die Zeit des 15. und frühen 16. Jahrhunderts. Die Quellen zur Geschichte der Mathematik im mitteleuropäischen Raum werden weiterhin ein Schwerpunkt meiner Arbeiten bleiben. Aber es trifft $\mathrm{zu}$, dass ich schon vor einiger Zeit begonnen habe, mich speziell auch mit Gauß und seinem Umfeld zu beschäftigen. In diesem Zusammenhang habe ich eine Datenbank der Briefe von und an Gauß erstellt unter Berücksichtigung der Vorarbeiten der maßgeblichen Gauß-Forscher. Diese Datenbank ist inzwischen online zugänglich ${ }^{1}$ und soll weiter ausgebaut werden. Auch die Bibliothek, die Gauß besessen hat, kann man jetzt aufgrund meiner Forschungen besser rekonstruieren als es vorher möglich war. Gauß’ Tätigkeit an der Universität Göttingen, seine Kontakte und Korrespondenzen werden mich auch weiterhin beschäftigen.

F: Welche Möglichkeiten einer späteren beruflichen Perspektive bieten sich einem jungen Menschen, der das Fach Geschichte der Naturwissenschaften gewählt hat?

A: Das ist eine Frage, die man so allgemein nur schwer beantworten kann. Mir scheint es in unserer Zeit besonders wichtig zu sein, zu zeigen und nachzuweisen, dass Naturwissenschaften und Technik eine unabdingbare historische Dimension haben, was immer wieder vernachlässigt wird, obwohl man 
schon seit Jahrzehnten von einer notwendigen Brücke zwischen beiden Kulturen spricht. Hier besitzt die Geschichte der Naturwissenschaften eine wichtige Funktion. Es hat sich gezeigt, dass Absolventen dieses Faches eine Reihe von Positionen an verschiedenen Institutionen erfolgreich besetzen konnten. Es können Museen, Archive oder auch Zeitschriftenredaktionen sein. Aus meiner Sicht ist es wichtig, dass sowohl in den Printmedien als auch in den neuen elektronischen Medien wissenschaftshistorisch kundige Personen tätig sind, damit ein breites Publikum besser informiert wird. Ich glaube, dass hier ein echter Bedarf besteht; dies zeigen auch Anfragen von verschiedenen Institutionen und Privatpersonen zu wissenschaftshistorischen Fragen, die oft im Zusammenhang mit neuesten wissenschaftlichen Erkenntnissen oder Pseudoerkenntnissen stehen. Um spektakuläre neue Erkenntnisse bewerten und einordnen zu können, ist es in vielen Fällen notwendig, über ein gründliches, solides Wissen im Bereich der Geschichte der Naturwissenschaften zu verfügen. Die vielen wissenschaftshistorischen Anfragen, die an uns herangetragen werden, beweisen, dass das Interesse am Fach lebendig ist, und ich denke, dass sich das in Zukunft noch verstärken wird.

F: Halten Sie vor dem Hintergrund dieses Interesses und der gesellschaftichen Bedeutung Ihres Faches die Einführung eines Bachelor-Studienganges für Geschichte der Naturwissenschaften für sinnvoll?

A: Im Prinzip ja. Die Frage ist nur, ob ein Bachelor für Geschichte der Naturwissenschaften Sinn macht oder ob man Kenntnisse in Geschichte der Naturwissenschaften in allgemeinere Bachelor-Studiengänge einfließen lassen sollte. Das wird ja auch in München diskutiert und ist vielleicht der erfolgreichere Weg, um den Studierenden verschiedener Fachrichtungen zu zeigen, dass die Geschichte der Naturwissenschaften Module liefern kann, um andere Studiengänge noch effektiver zu gestalten. Hier ist München durch das Deutsche Museum mit seinen Sammlungen und der Möglichkeit, Praktika zu absolvieren, ein besonders gut geeigneter Ort. Wir haben eine Reihe von Vorschlägen gemacht, wie man die Geschichte der Naturwissenschaften in andere Bachelor-Studiengänge integrieren kann. Noch wichtiger wäre es, auch einen Master-Studiengang für Geschichte der Naturwissenschaften zu bekommen - auch der ist in München in Planung -, und ich denke, dass man Studierende, die unterschiedliche Bachelor-Abschlüsse erworben haben, davon überzeugen kann, sich intensiver mit der Geschichte der Naturwissenschaften zu beschäftigen, um später Einsatzmöglichkeiten in verschiedenen Berufsfeldern zu finden.

F: Sie sind Mitglied einer Reihe von nationalen und internationalen Akademien und Gesellschaften und waren als Vertreter Ihres Faches auch im internationalen Rahmen tätig. Welche wissenschaftsorganisatorischen und wissenschaftspolitischen Tätigkeiten zählen Sie zu den wichtigsten in Ihrem Leben? 
A: In den 1980er Jahren war ich als Fachgutachter der DFG tätig. Als Gutachter konnte ich die Entwicklung nicht nur beobachten, sondern auch ein wenig beeinflussen. Damals gab es die Sicherheit, dass Projekte, die sehr positiv begutachtet worden waren, auch genehmigt wurden. Vor kurzem habe ich aber erleben müssen, dass dies inzwischen offenbar nicht mehr zutrifft: Ein Projekt, das von den Fachgutachtern als absolut förderungswürdig eingeschätzt worden war, wurde vom Fachkollegium mit der Begründung abgelehnt, man vermisse „spannende Arbeitshypothesen“. Für wichtig halte ich auch meine Tätigkeit im Vorstand einer unserer beiden Fachgesellschaften, der Deutschen Gesellschaft für Geschichte der Medizin, Naturwissenschaft und Technik (DGGMNT). Dort war ich drei Jahre lang stellvertretender Vorsitzender und drei Jahre lang Vorsitzender. Seit 1989 bin ich Mitglied der Deutschen Akademie der Naturforscher Leopoldina, die vor kurzem zur Nationalakademie erhoben worden ist. Acht Jahre lang war ich dort als Obmann für die Wissenschafts- und Medizingeschichte verantwortlich und gehörte auch dem Senat an. Im internationalen Bereich war es für mich sehr wichtig, dass ich schon 1971 die Gelegenheit hatte, den 13. Internationalen Kongress für Geschichte der Naturwissenschaften in Moskau zu besuchen. Bis zum Jahr 2001 habe ich dann an allen weiteren internationalen Kongressen dieser Art teilgenommen - häufig als Vortragender und als Vertreter der Bundesrepublik Deutschland. Bis heute bin ich Mitglied im Nationalkomitee der Bundesrepublik Deutschland für Geschichte der Naturwissenschaften und konnte so - über die internationale Vernetzung der Wissenschaftsgeschichte - mein eigenes Wissen in die internationale Community einzubringen. Seit vielen Jahren gehöre ich der International Commission on the History of Mathematics an, deren Treasurer ich seit 1990 bin. Auch dort war es mir möglich, die internationale Entwicklung des Fachs zu verfolgen und zu einem Teil mit zu beeinflussen.

Das Interview führte Andreas Kühne (München) am 22. April 2008.

\section{Anmerkung}

1 Gauss.gwi.uni-muenchen.de

\section{Nachbemerkung der Redaktion:}

Aus München erreicht uns die überraschende Nachricht, dass die Ludwig-Maximilians-Universität München die Professur von Menso Folkerts trotz längerfristiger entsprechender Vorbereitungen nach seinem Wechsel in den Ruhestand nicht neu ausschreiben will. Hintergrund ist der sog. 
50/40/10-Prozess der LMU, wonach jeder Fachbereich dazu verpflichtet ist, $10 \%$ der Professuren abzugeben und über 40\% der Professuren mit dem Präsidium Verhandlungen über Neuausschreibungen zu führen. Für die Wissenschaftsgeschichte wäre der Wegfall dieser Professur ein herber Verlust - zumal München bislang einer der bedeutendsten deutschen Standorte des Faches war. Es ist kaum zu verstehen, wie eine mit dem Prädikat der Exzellenz ausgezeichnete Universität angesichts der bisherigen Evaluationen der Münchner Wissenschaftslandschaft eine Eckprofessur in einem innovativen Wissenschaftsfeld auf der Nahtstelle zwischen den Natur- und Geisteswissenschaften aufgeben kann. Zwei Perspektiven geben Anlass zur Hoffnung, dass die Wissenschaftsgeschichte in München mit verändertem Profil weitergeführt werden kann. Erstens hat die Universitätsleitung signalisiert, dass eine Wiederzuweisung der Professur als W2 möglich sei. Zweitens werden die Kolleginnen und Kollegen des Münchner Zentrums für Wissenschaftsund Technikgeschichte im Rahmen eines LMU-internen Wettbewerbs um die Bildung neuer Zentren ein im Kern wissenschaftshistorisch orientiertes Programm unter Einbindung benachbarter Fachbereiche ausarbeiten. 